\title{
Application of Active Thermography for mode II delamination detection in CFRP: comparison between modalities of the technique and a numerical modelling study of defect parameters' influence
}

\author{
by M. Grosso*, H. G. Kotik** and G. R. Pereira**
}

* Laboratory of Non Destructive Testing, Corrosion and Welding (LNDC), Department of Metallurgical and Materials Engineering, Federal University of Rio de de Janeiro, Rio de Janeiro, RJ, Brazil, marcellag@metalmat.ufrj.br

** Composite Materials Laboratory (LaCom), Department of Metallurgical and Materials Engineering, PEMM COPPE/UFRJ - Federal University of Rio de Janeiro, CEP 21941-972, Rio de Janeiro, RJ, Brazil, hectorkotik@metalmat.ufrj.br

${ }^{* * \star}$ Department of Metallurgical and Materials Engineering, COPPE/UFRJ - Federal University of Rio de Janeiro, CEP 21941-972, Rio de Janeiro, RJ, Brazil, gpereira@metalmat.ufrj.br

\begin{abstract}
In this study, three modalities of Thermography (Pulsed, Pulsed Phase and Lockin) were used to detect delamination defects in CFRP samples from the 4ENF test. The results obtained for each modality were compared in relation to the real delamination area in these samples. In addition to the experimental analysis, a computational simulation model was also created to evaluate the influence of defect parameters (depth and thickness) on its thermal contrast and thus estimate the detection limit presented by the Pulsed modality.
\end{abstract}

\section{Introduction}

As the application of CFRP (Carbon Fiber Reinforced Polymer) has increased in many industries, especially in aircraft manufacturing industry and automotive industry, there has also been a growing demand for non-destructive techniques and their development for monitoring, evaluating and ensuring the integrity of these materials. Defects may be caused by cyclic stress, impact damages and any other anomaly arising during the material manufacturing process and their presence lead to a significant reduction of the load capacity and degradation of mechanical properties. Among the many mechanics that can cause damage to laminated composites, loads inducing interlaminar fracture (delamination) by mode II are frequent defects. The use of non-destructive techniques becomes a useful tool for guarantee structural integrity and estimate the risks involved when a defect is presented in the material.

The four-point bending test employing end notched specimens (4ENF) is a commonly used test to estimate the value of mode II fracture toughness. The length of the generated interlaminar crack is an important parameter to be measured because it can be correlated with the strain energy release rate [1]. For the classical method, the toughness calculation considers only the crack length on sample lateral faces, however, there is a possibility of crack tunnelling and propagation to different extents at different points of the sample width. Thus, the purpose of this work is to evaluate the ability of the three most used modalities of active thermography technique to detect delamination area in CFRP samples submitted to fracture tests to generate a mode II interlaminar crack.

For this study, the Pulsed, Pulsed Phase and Lockin Thermography were evaluated both for detection of delamination present in the samples and to investigate the real delamination area. The Pulsed Thermography consists of briefly heating the sample surface and, then, the surface temperature decay is recorded. The result of the inspection using this modality is presented as a sequence of thermograms. In Pulsed Phase Thermography, the temperature evolution over time obtained in Pulsed modality is converted to the frequency domain through the Fourier Transform (FFT) and the result is expressed in two images, one referring to the values of angle of phase and the other concerning the amplitude (Fourier module) for each pixel at the chosen frequency analysis. The Lockin modality consists of the periodic heating of the surface of the material through modulated lamps and simultaneously the temperature evolution is monitored along the heating cycles. For each test, it is necessary to choose a single frequency of modulation and the choice of this is depends on the depth of penetration of the thermal wave desired in the material. The result of the Lockin is presented in the form of two images, one referring to the phase angle and the other referring to the amplitude, both generated by Fourier transform $[2,3,4]$. The results obtained by each technique were compared in order to evaluate the resolution obtained in the area of defects.

In addition to experimental analysis, a computational simulation model based on finite element methods was developed in order to reproduce the 3-dimensional physical phenomena during the Pulsed Thermography inspection in these materials. This model has become a useful tool to evaluate the influence of some geometric parameters related to the delamination defect area (thickness, depth and crack position) with the thermal contrast value obtained. 


\section{Material and Methods}

\subsection{Material}

For this study, 3 samples were evaluated. All samples were made of carbon-fiber reinforced plastic (CFRP) using epoxy Wilpox CH4L [5] as matrix and carbon fiber UD Torayca ${ }^{\circledR}$ T300 as reinforcement. The composite was manufactured by the hand lay-up method using a vacuum bag and a lay-up [0]14 (unidirectional). In order to induce the discontinuity (notch), a $25 \mu \mathrm{m}$ separating film was inserted at half the thickness (between layers 7 and 8 ) of the samples during the hand lay-up process. At the end of the manufacturing process, in each sample there was a film whose length was approximately $55 \mathrm{~mm}$ from one of its edges.

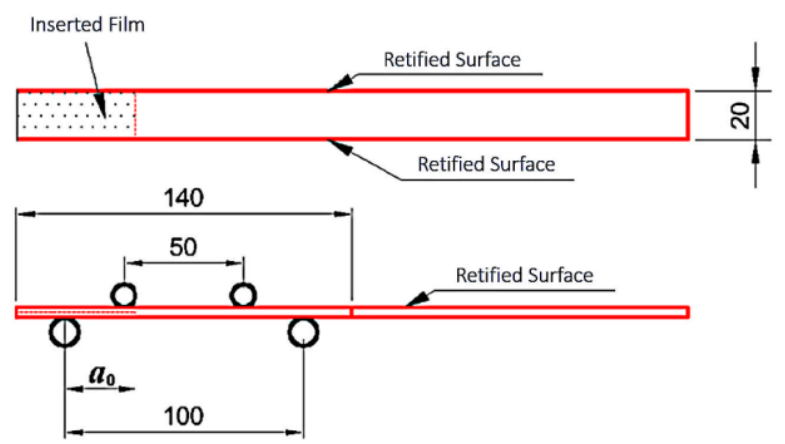

Fig. 1. CFRP sample with a $25 \mu \mathrm{m}$ separating film inserted at half the thickness [6].

Table 1. Geometric parameters of the samples and defects for the experimental tests

\begin{tabular}{|c|c|c|c|c|c|}
\hline Sample & Width $(\mathrm{mm})$ & Length $(\mathrm{mm})$ & $\begin{array}{c}\text { Thickness } \\
(\mathrm{mm})\end{array}$ & $\begin{array}{c}\text { Crack length } \\
\text { (transversal } \\
\text { side 1, } \mathrm{mm})\end{array}$ & $\begin{array}{c}\text { Crack length } \\
\text { (transversal } \\
\text { side 2, mm) }\end{array}$ \\
\hline 1 & 19,48 & 280 & 3,717 & 85,5 & 82,0 \\
\hline 2 & 20,25 & 280 & 4,261 & 68,0 & 68,0 \\
\hline 3 & 18,87 & 280 & 4,482 & 62,0 & 62,0 \\
\hline
\end{tabular}

\subsection{Experimental Setup - Mechanical Test}

The 4ENF tests were performed in a universal testing machine EMIC of $20 \mathrm{kN}$ capacity according to the guidelines of ESIS [7]. The interlaminar crack growth was monitored with two USB digital microscopes, one on each side of the specimen using 50X magnification [6]. Based on the linear elastic fracture mechanics theory and employing load measurement data and compliance results exposed by Kotik [6], the values of mode II interlaminar fracture toughness expressed by the strain energy release rate $\left(G_{\|}\right)$can be estimated from the values of the delaminated area obtained. Assuming a constant compliance change rate related to the crack area ( $\boldsymbol{d} \boldsymbol{C} / \boldsymbol{d} \boldsymbol{A}$ is constant for this case), this calculation can be made employing the initial and final values of the crack area and compliance, as exposed in Eq.1and Eq.2.

$$
\begin{gathered}
G_{I I}=\frac{P^{2}}{2} \frac{d C}{d A} \\
G_{I I} \approx \frac{P^{2}}{2} \frac{C_{f}-C_{0}}{A_{f}-A_{0}}
\end{gathered}
$$

From the analysis of the above equations, the importance of dimensioning the defective area is evidenced, since it influences in toughness measurement. Therefore, the evaluation of a non-destructive technique (thermography in the 
case of this work) capable of estimating the defective area with the minimum uncertainties becomes a very useful tool for the correct calculation of toughness.

\subsection{Experimental Setup - Thermographic Tests}

All tests were carried out with an infrared camera, which is an uncooled microbolometer Focal Plane Array (FPA) camera and a set of $3,4 \mathrm{~kW}$ halogens lamps was used to generate the heat pulse on the material surface (reflection mode). The camera was controlled by a computer which allowed the capturing of thermal images with a frame rate of $30 \mathrm{~Hz}$. In Pulsed Thermography test, after the $10 \mathrm{~s}$ of heating phase, the camera collected the frames for $50 \mathrm{~s}$ of the subsequent cooling. For the Pulsed Phase Thermography (PPT) the thermograms sequence (temperature evolution over time) obtained in PT modality was converted to the frequency domain through the Fourier Transform (FFT) and the result is expressed in values of phase angle of phase and amplitude for each pixel. In Lockin tests, 5 modulation cycles were used by each test, totalling 1000 thermograms. The frequencies evaluated in Lockin tests were $0.1,0.05$ and $0.025 \mathrm{~Hz}$. Although the defects were positioned at half the thickness of the samples, the two surfaces (Retified and non-retified) of each sample were inspected in all evaluated modalities.

\subsection{Computational simulation model}

To reproduce the physical phenomena that occur during the Pulsed Thermography tests, a Comsol Multiphysics $\AA$ software, version 5.1, was used to create the simulation model and the default physical model heat transfer in solid module was used. A virtual solid was created to represent the $3 \mathrm{D}$ geometry of the sample used in the experimental tests of this work with the corresponding inserted defects and their dimensions.

In this model, to calculate the temperature behavior of the heat transfer principle through conduction in transient state, the Eq. 3 was used:

$$
\rho C_{p} \frac{\partial T}{\partial t}=\nabla \cdot(k \nabla T)+Q
$$

where $\rho\left(\frac{\mathrm{kg}}{\mathrm{m}^{3}}\right)$ is density, $C_{p}\left(\frac{\mathrm{J}}{\mathrm{kg} \cdot \mathrm{m}}\right)$ is the heat capacity at constant pressure, $\mathrm{T}(\mathrm{K})$ is the temperature, $\mathrm{t}(\mathrm{s})$ is the time elapsed during the test, $k\left(\frac{W}{m} \cdot K\right)$ is the material thermal conductivity and $Q\left(\frac{W}{m^{2}}\right)$ is the energy incident on the surface of the sample by the heat source.

Two boundaries conditions was added in this model to reproduce the emission of infrared radiation issued by the surface material, Eq. 4, and the convection cooling of the surface of the material, Eq. 5, as described below:

$$
\begin{aligned}
& -n \cdot(-k \nabla T)=\varepsilon \sigma\left(T_{a m b}^{4}-T^{4}\right) \\
& -n \cdot(-k \nabla T)=h \cdot\left(T_{a m b}^{4}-T^{4}\right)
\end{aligned}
$$

where $\varepsilon$ is the emissivity, $\sigma\left(\frac{W}{m^{2} \cdot K^{4}}\right)$ is the Stefan-Boltzmann constant and $h\left(\frac{W}{m^{2} \cdot K}\right)$ is the constant convective heat transfer coefficient in the air.

In all cases, the values of each variable and physical properties contained in Eqs (1), (2) and (3) were given as parameters in the global definition of the model in the software. From the software's material library, two materials were used to reproduce the sample: air (delamination defect) and Carbon fiber (entire sample). The mesh used was chosen according the control physics and with finer size.

\section{Results}

\subsection{Thermographic tests}

Fig. 2 shows the results obtained on both surfaces evaluated for sample 1. Analysing these results, it is possible to observe both in thermogram obtained by PT (Fig.2(a)) and in the phase image obtained by Lockin (Fig.2(c)) a region closer to the centre of the sample where there is a change in the parameters illustrated in the images. According to the crack measurement performed on the transverse surface of the sample by visual inspection (Table 1), such behaviour may 
be related to the change from a region of the sample where the crack is present (left) to a region where there is no defect (right).

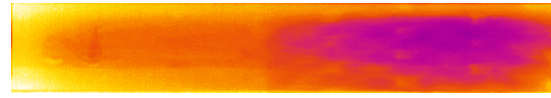

(a)

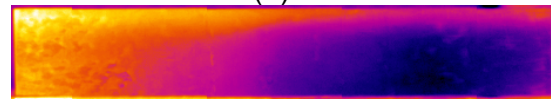

(d)



(b)

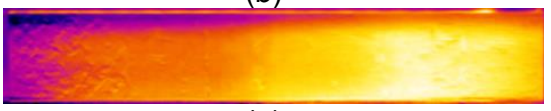

(e)



(c)

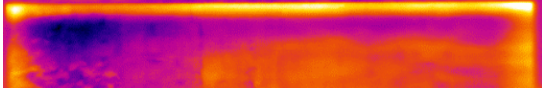

(f)

Fig. 2. Sample 1: Retified surface (a) Raw thermogram obtained by PT, (b) phase image obtained by PPT, (c) phase image at $0.05 \mathrm{~Hz}$ by Lockin; Non-retified surface (d) raw thermogram obtained by $P T$, (e) phase image obtained by $P P T$, (c) phase image at $0.05 \mathrm{~Hz}$ by Lockin.

The results obtained by the three modalities evaluated in this study for sample 2 (Fig. 3) illustrate a change in behaviour throughout the sample, where it becomes possible to observe a transition line closer to the centre of the sample. As in the previous sample, this transition line is located close to the final crack length measured by the cross section. It is also important to note that the transition region has become more evident in the results obtained in the inspection on the retified surface (Fig. 3(a)-(c)).

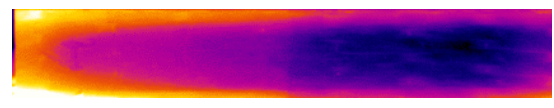

(a)



(d)

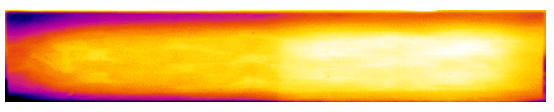

(b)

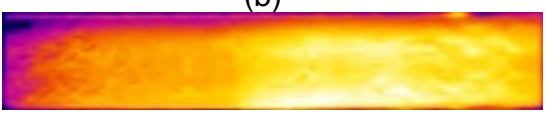

(e)

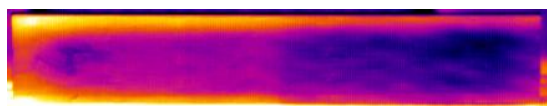

(c)

(f)

Fig. 3. Sample 2: Retified surface (a) Raw thermogram obtained by PT, (b) phase image obtained by PPT, (c) phase image at $0.05 \mathrm{~Hz}$ by Lockin; Non-retified surface (d) raw thermogram obtained by PT, (e) phase image obtained by PPT, (c) phase image at $0.05 \mathrm{~Hz}$ by Lockin.

For the sample 3, as in the previous samples, there is a transition behaviour between the region where the crack is present (left) to the region without defect (right). This transition becomes more abrupt in the region close to the centre of the sample and which corresponds to the end in the crack length observed in the cross section of the sample.

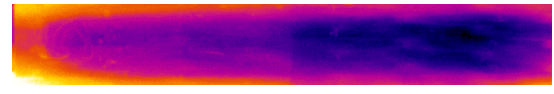

(a)

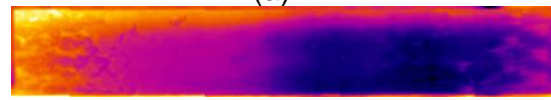

(d)

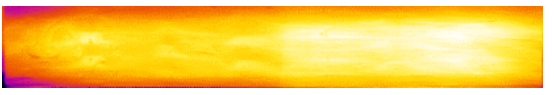

(b)



(e)



(c)

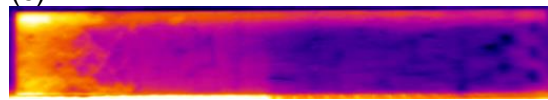

(f)

Fig. 4. Sample 3: Retified surface (a) Raw thermogram obtained by $P T$, (b) phase image obtained by PPT, (c) phase image at $0.05 \mathrm{~Hz}$ by Lockin; Non-retified surface (d) raw thermogram obtained by PT, (e) phase image obtained by PPT, (c) phase image at $0.05 \mathrm{~Hz}$ by Lockin.

From the results above, it was possible to observe a change in thermal behaviour related to the delamination areas in the samples. Thus, the evaluated modalities of active thermography proved to be potential tool to detect delamination defects that cannot be detected by visual inspection on the surface of the samples. However, comparing the results obtained between the modalities regarding the definition, resolution and reproducibility in the defective areas, PPT and Lockin proved to be more effective. The choice of which of these two modalities becomes more appropriate must also take into account the advantages and disadvantages of each one. PPT is a faster method considering that in just one test, it becomes possible to evaluate several frequencies, which for Lockin is restricted to the analysis of a single frequency per test. However, for Lockin, the delamination depth estimate can be calculated more easily than a PPT due to the choice of modulation frequency.

Another important aspect that should be highlighted is that, due to the results obtained, the modalities were able to identify the defective areas located at a depth of approximately $2 \mathrm{~mm}$ (half the thickness of the sample). There are very 
few studies in the literature for the inspection of CFRP materials that evaluate defects at depths close to those studied in this work.

\subsection{Simulation results and quantitative analysis}

In addition to the experimental analysis, this work aims to evaluate the influence of some geometrical aspects of the defects in the detection capacity presented by the Pulsed Thermography through computational simulation model. The influence of the effect of the defect depth on its thermal contrast was evaluated in 3 conditions: defects positioned at $1 / 4$ of the sample thickness (depth $=1.05 \mathrm{~mm}$ ), at $1 / 2$ of sample thickness $($ depth $=2.10 \mathrm{~mm}$ ) and $3 / 4$ of the thickness $($ depth $=$ $3.15 \mathrm{~mm}$ ). For each condition, the influence of the defect thickness was also evaluated. The chosen values were: $0.8 \mathrm{~mm}$, $0.4 \mathrm{~mm}, 0.2 \mathrm{~mm}, 0.1 \mathrm{~mm}$ and $0.05 \mathrm{~mm}$. As described in item 2.1, in order to induce the discontinuity (notch)in the experimental tests, a $25 \mu \mathrm{m}$ separating film was inserted at half the thickness. In the simulations, it was decided to position the representative defect of delamination in the central region of the sample, as it is considered that this condition is more critical for its detection, since it would not be possible to detect it by visual inspection on the two ends of the sample (larger axis).

As in the experimental tests, the result obtained from this computational simulation model is presented in the form of images illustrating the thermal distribution on the surface of the material as a function of time and also curves of temperature evolution over time for material regions selected by the user. Fig. 3(a) shows the image with the temperature distribution on the sample surface obtained by the computational simulation model proposed in this study and Fig. 3(b) illustrates the probe positions on the surface of the sample to obtain the temperature evolution curves over time (Fig. 3(c)).

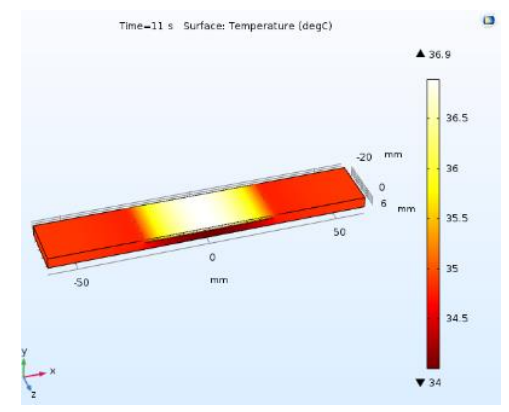

(a)

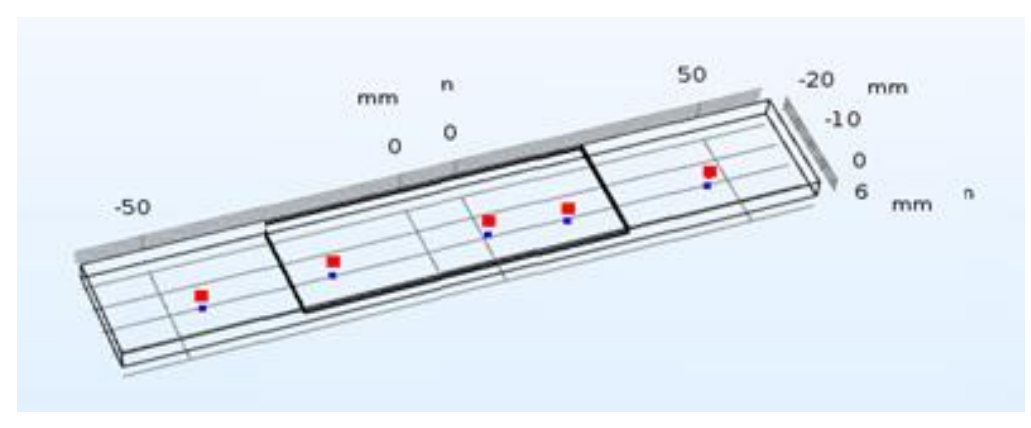

(b)



(c)

Fig. 3. (a) Image illustrating the thermal distribution on the surface of the material at $11 \mathrm{~s}$, (b) position of probes for temperature measurement in defective and non-defective regions and (c) the temperature evolution curves corresponding to each probe.

The thermal contrast data instead of temperature curves will prove more helpful in extracting the defect information, such as depth. Thermal contrast is a better feature to use as an input data as it highlights the difference in 
temperature between the defective and non-defective areas. The absolute thermal contrast can be calculated as described in Eq. 4:

$$
T_{c}(t)=T_{d}(t)-T_{s}(t)
$$

where $T_{c}(t)$ is the absolute thermal contrast, $T_{d}(t)$ is the temperature on the defective area and $T_{s}(t)$ is the temperature on the sound (non-defective) area.

Fig.4 shows the graphs of absolute thermal contrast for each of the simulated depths of the defects. For each depth, the influence of defect thickness was also evaluated. Analysing these results, it is observed that there is a reduction in thermal contrast due to the increase in the defects' depth. Although such behaviour can be correlated to the theory of thermal waves, the knowledge of the magnitude of the contrast in each of these conditions evaluated through computational simulation allows us to estimate that delamination defect type could be detected by Pulsed Thermography to depths up to $2.10 \mathrm{~mm}$. Defects located at greater depths have thermal contrast values so low that their detection is limited to the sensitivity of the thermal camera used.

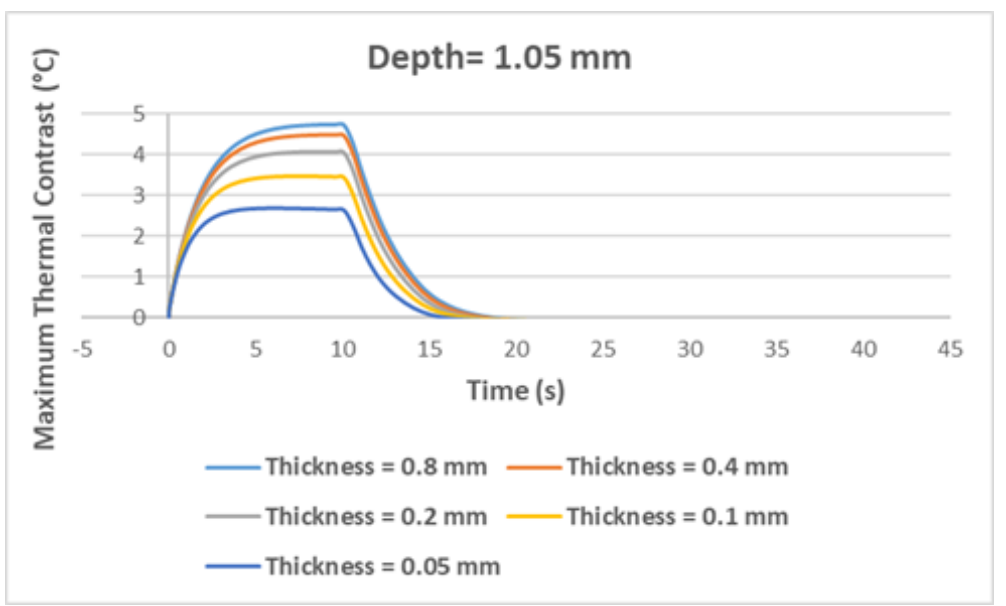

Fig. 4. Graph illustrating the absolute thermal contrast obtained for the defects at $1.05 \mathrm{~mm}$ depth.

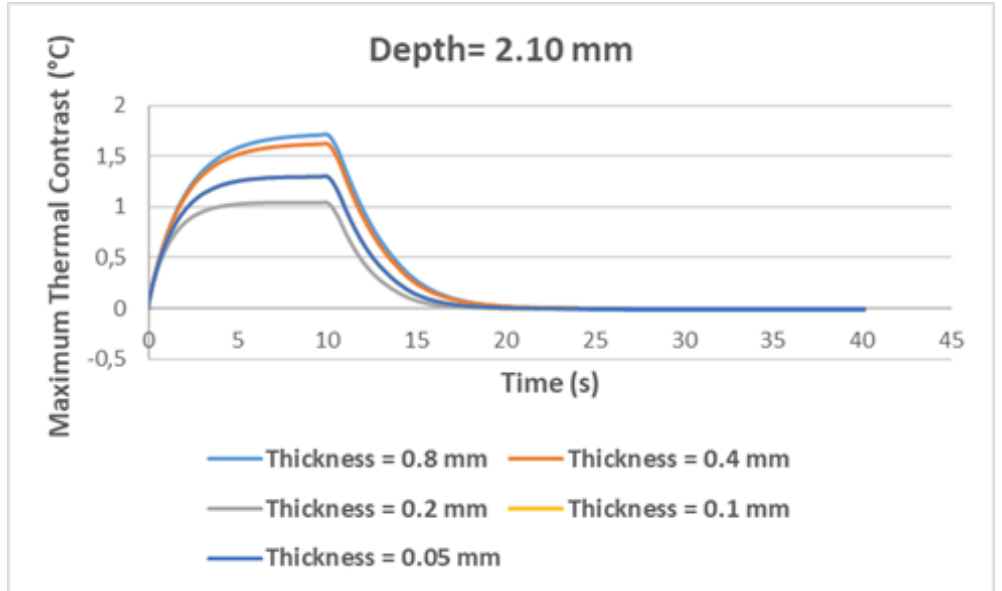

Fig. 5. Graph illustrating the absolute thermal contrast obtained for the defects at $2.10 \mathrm{~mm}$ depth. 


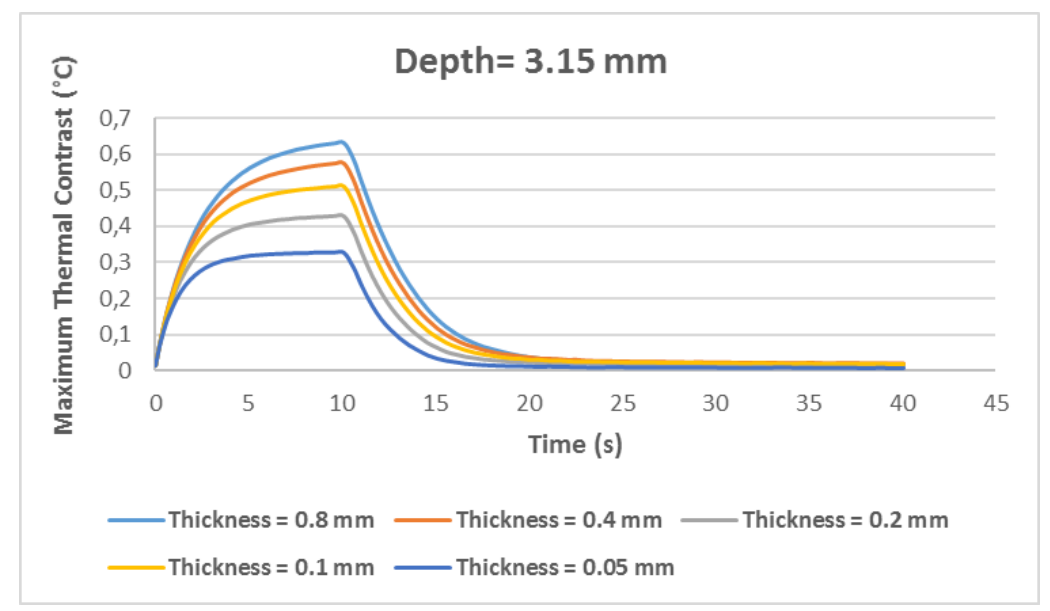

Fig. 6. Graph illustrating the absolute thermal contrast obtained for the defects at $3.15 \mathrm{~mm}$ depth.

In addition to the analysis related to the influence of the depth of the defects, it was considered important to also analyze the magnitude of the influence of the thickness in the maximum thermal contrast. The knowledge of the magnitude of the thermal contrast allows us to estimate whether such defect, according to its thickness and depth, can be detected or not by the modality. According to the results obtained (Fig. 7), it is observed that all defects evaluated at a depth of 1.05 $\mathrm{mm}$ could be detected, however, for a depth of $2.10 \mathrm{~mm}$, defects of thickness less than $0.025 \mathrm{~mm}$ by the trend would not be detected due to its low thermal contrast value. As described in item 2.1, a film was inserted at half the material' thickness to induce the interlaminar crack propagation during the 4ENF test. According to the data obtained by simulation, disregarding the increase in the delamination area and the thickness as a result of the 4ENF test and considering only the $25 \mu \mathrm{m}$ separating film thickness (most critical condition for detection due the smallest thickness), the Pulsed modality would present a thermal contrast that would allow the detection of this type of defect at depths up to $2.10 \mathrm{~mm}$.

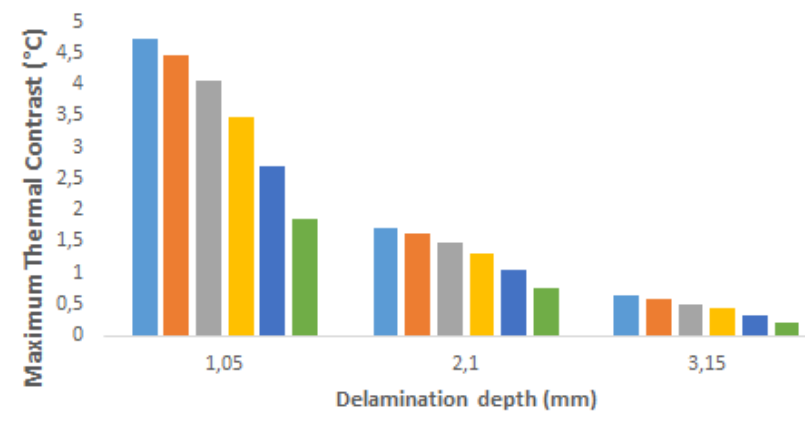

Thickness $(\mathrm{mm})=0,8=0,4=0,2=0,1 \backsim 0,05 \backsim 0,025$

Fig. 7. Graph comparing the maximum absolute thermal contrast for the defects in each depth simulated, considering all thicknesses evaluated.

\section{Conclusion}

In this work, three most used modalities of active thermography technique (Pulsed, Pulsed Phase and Lockin) were evaluated to detect delamination in CFRP samples submitted to fracture tests to generate a mode II interlaminar crack. According to the experimental results, it was possible to detect the region in which the delamination was present. However, considering that for a correct measurement of the mode II interlaminar fracture toughness it is necessary to calculate the delaminated area, the Pulsed Phase and Lockin were more suitable for this purpose due to their better definition on the defective area. From the images generated by these modalities, techniques of image post-processing and automatic pattern recognition can assist in the calculation of the defective area in a later stage of continuing this work.

The results obtained by the computer simulation allowed the knowledge of the influence of the depth and thickness of the defects in their thermal contrast, which makes it possible to estimate the detection limit presented by the Pulsed 
modality for this type of defect. Through the magnitude estimated by the simulation, delamination defects whose thickness is greater than $0.025 \mathrm{~mm}$ and positioned at depths up to $2.1 \mathrm{~mm}$ in this material could be detected. For a more accurate estimate of the detection limit as a function of the defect parameters, we intend to continue this study using mathematical and artificial intelligence algorithms.

\section{REFERENCES}

[1] B. R. K. Blackman, A. Pavan and J. G. Williams, Fracture of Polymers, Composites and Adhesives II, Boston: Elsevier, 2003.

[2] Maldague, X.P.V., 'Infrared and Thermal Testing - non destructive testing', ASNT; 2001.

[3] Almond, D. and Pickering, S. 'Matched excitation energy comparison of the pulse and lock-in thermography NDE techniques', NDE\&E International, 41 (2008) 501-509.

[4] Busse, G. and Wu, D. 'Lock-in thermography for nondestructive evaluation of materials', Revue Generale de Thermique, 37 (2008) 501-509.

[5] Carmas Composites, "Informe técnico: Wilpox APV CH4L," 2016.

[6] H. G. Kotik, "Fractura y fatiga de materiales compuestos de matriz polimérica empleados en palas de generadores eólicos de alta potencia.," Bahia Blanca, Argentina, 2016.

[7] Davies P, Blackman BRK, Brunner AJ. Mode II Delamination. In: Moore DR, Pavan A, Williams JG, editors. ESIS Publ. 28 Fract. Mech. Test. methods Polym. Adhes. Compos. 1st ed., Oxford: Elsevier Science Ltd.; 2001, p. 307-16. 